\title{
Solubility of Anthraquinone Derivatives in Supercritical Carbon Dioxide: New Correlations
}

\author{
Ratna Surya Alwi $^{1, * \mathbb{D}}$, Chandrasekhar Garlapati ${ }^{2} \mathbb{D}$ and Kazuhiro Tamura ${ }^{3}$ \\ 1 Department of Chemical Engineering, Fajar University, Makassar 90231, Indonesia \\ 2 Department of Chemical Engineering, Puducherry Technological University (Formerly Known as \\ Pondicherry Engineering College), Puducherry 605014, India; chandrasekar@pec.edu \\ 3 Division of Natural System, Graduate School of Natural Science and Technology, Kanazawa University, \\ Kakuma-machi, Kanazawa 920-1192, Japan; tamura@se.kanazawa-u.ac.jp \\ * Correspondence: ratnasya@gmail.com; Tel.: +62-8114-480-880
}

check for updates

Citation: Alwi, R.S.; Garlapati, C.;

Tamura, K. Solubility of

Anthraquinone Derivatives in

Supercritical Carbon Dioxide: New

Correlations. Molecules 2021, 26, 460.

https://doi.org/10.3390/

molecules 26020460

Academic Editors: Mauro Banchero

and Barbara Onida

Received: 8 December 2020

Accepted: 12 January 2021

Published: 17 January 2021

Publisher's Note: MDPI stays neutral with regard to jurisdictional clai$\mathrm{ms}$ in published maps and institutional affiliations.

Copyright: $\odot 2021$ by the authors. Licensee MDPI, Basel, Switzerland. This article is an open access article distributed under the terms and conditions of the Creative Commons Attribution (CC BY) license (https:// creativecommons.org/licenses/by/ $4.0 /)$.

\begin{abstract}
Solubility of several anthraquinone derivatives in supercritical carbon dioxide was readily available in the literature, but correcting ability of the existing models was poor. Therefore, in this work, two new models have been developed for better correlation based on solid-liquid phase equilibria. The new model has five adjustable parameters correlating the solubility isotherms as a function of temperature. The accuracy of the proposed models was evaluated by correlating 25 binary systems. The proposed models observed provide the best overall correlations. The overall deviation between the experimental and the correlated results was less than $11.46 \%$ in averaged absolute relative deviation (AARD). Moreover, exiting solubility models were also evaluated for all the compounds for the comparison purpose.
\end{abstract}

Keywords: solubility; supercritical carbon dioxide; anthraquinone; AIC; new correlations

\section{Introduction}

Supercritical fluid (SCF) applications in process industry have gained a lot of momentum. The proper application solely depends on exact information on solubility, therefore, the estimation of solubility of a variety of substances in supercritical fluids has taking place in recent literature [1]. Among various supercritical fluids, carbon dioxide is one that has more attention due to its interesting and easily attainable critical properties [1]. Dyeing industry and pharmaceutical industry require solubility data, but the data are limited and available at particular specified temperatures and pressures [1]. Measuring solubility at each and every point would be a tedious task, therefore modeling is a must [2]. The present study is concerned about the modeling of anthraquinone derivatives in supercritical carbon dioxide. Anthraquinone derivatives are majorly used in dyeing industries-the exact prediction of solubility data are very much essential for the development of supercritical dyeing process. There are five frameworks through which solubility data are analyzed [3]. Out of five approaches, thermodynamic frameworks based on solid-gas equilibrium criteria and solid-liquid equilibrium criteria are very successful [4]. Solid-gas equilibrium approach requires critical, chemical, and physical information for the modeling. The availability of such information is very rare; therefore, solid-gas equilibrium approach entirely depends on group contribution methods for those necessary properties. Therefore, the solid-gas equilibrium approach would be purely lies on the accuracy of the predicted properties. Sometimes these properties may not be real and the corresponding correlation may not be appropriate. Therefore, we need to look for an alternative correlating approach under thermodynamic framework for better correlation purpose; under such circumstance, the solid-liquid equilibrium criteria approach may be useful in correlating the solubility [5-7]. In the present work we aimed at the development of new solubility models for the anthraquinone derivative which will be useful for supercritical dyeing process. 
In this work, we proposed two new models based on solid-liquid equilibrium criteria. Further, important exiting solubility models are also evaluated for all the compounds for the comparison purpose. The following section deals with existing solubility models considered in this study.

\section{Existing Solubility Models}

2.1. Empirical Models

2.1.1. Chrastil Model

Chrastil et al. [8] proposed a semi empirical model based on solvate complex theory and have related the solubility of solute to density of supercritical fluid as follows:

$$
S_{2}=\rho_{\mathrm{ScCO}_{2}}^{k} \exp \left(\frac{A_{1}}{T}+A_{2}\right)
$$

where $S_{2}$ is the solute solubility in $\mathrm{kg} \cdot \mathrm{m}^{-3}, k$ is the association number, $d_{1}$ is constant, and $d_{2}$ presents the function of enthalpy of solvation and vaporization. Equation (1) can be rewritten [9] to be mole fraction terms as follows:

$$
y_{2}=\frac{\left(\rho_{\mathrm{ScCO}_{2}}\right)^{k-1} \exp \left(\frac{A_{1}}{T}+d_{2}\right)}{\left[1+\left(\rho_{\mathrm{ScCO}_{2}}\right)^{k-1} \exp \left(\frac{A_{1}}{T}+A_{2}\right)\right]}
$$

\subsubsection{Adachi and Lu Model}

Adachi and Lu (1983) [10] modified Chrastil's equation by considering the quantity $k$ to be density-dependent and the model can be written as:

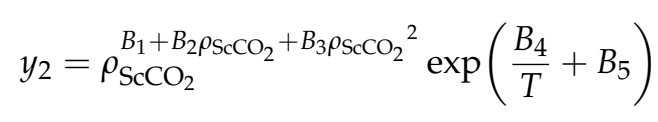

where $y_{2}$ is the solute solubility in the mole fraction, the $B_{1}$ to $B_{5}$ are parameters constant.

\subsubsection{Mitra-Wilson Model}

Mitra and Wilson (1991) [11] developed an empirical model for solubility of solute as a function of temperature and pressure:

$$
\ln S_{2}=C_{1} \ln P+C_{2} T+C_{3} P T+C_{4} \frac{P}{T}+C_{5}
$$

Here, $P$ is the pressure system used in atm, and $C_{1}$ to $C_{5}$ are the constant parameters.

\subsubsection{Keshmiri Model}

Keshmiri et al. (2014) [12] proposed the possible linear relationship between $\ln y_{2}$ and $\ln \rho_{\mathrm{ScCO}_{2}}$ as the following expression:

$$
\ln y_{2}=D_{1}+\frac{D_{2}}{T}+D_{3} P^{2}+\left(D_{4}+\frac{D_{5}}{T}\right) \ln \rho_{\mathrm{ScCO}_{2}}
$$

where $T$ and $P$ are the temperature and pressure system used, respectively. The $D_{1}$ to $D_{5}$ are constant parameters.

\subsubsection{Khansary Model}

Subsequently, Khansary et al. (2015) [13] also developed a model relationship between $\ln y_{2}$ and $\ln \rho_{\mathrm{ScCO}_{2}}$ as:

$$
\ln y_{2}=\frac{E_{1}}{T}+E_{2} P+E_{3} \frac{P^{2}}{T}+\left(E_{4}+E_{5} P\right) \ln \rho_{\mathrm{ScCO}_{2}}
$$


The $E_{1}$ to $E_{5}$ are constant parameters.

\subsubsection{Bian Model}

Bian et al. (2016) [14] found a model with five constant parameters with relationship between solubility of solute $\left(y_{2}\right)$ in mole fraction and density, $\rho_{\mathrm{ScCO}_{2}}$, and obtained the following model:

$$
\ln y_{2}=F_{1}+\frac{F_{2}}{T}+\frac{F_{3} \rho_{\mathrm{ScCO}_{2}}}{T}+\left(F_{4}+F_{5} \rho_{\mathrm{ScCO}_{2}}\right) \ln \rho_{\mathrm{ScCO}_{2}}
$$

where $F_{1}$ to $F_{5}$ are the model parameters.

\subsubsection{Garlapati and Madras Model}

Garlapati and Madras [2] proposed an empirical model and related solute solubility to density of supercritical fluid as:

$$
\ln y_{2}=G_{1}+\left(G_{2}+G_{3} \rho_{\mathrm{ScCO}_{2}}\right) \ln \rho_{\mathrm{ScCO}_{2}}+\frac{G_{4}}{T}+G_{5} \ln \rho_{\mathrm{ScCO}_{2}} T
$$

where $G_{1}$ to $G_{5}$ are constant parameters.

\subsubsection{Reddy Model}

Reddy et al. (2018) [15] proposed an empirical model based on degrees of freedom analysis as:

$$
y_{2}=\left(H_{1}+H_{2} P_{r}\right) T_{r}^{2}+\left(H_{3}+H_{4} P_{r}\right) T_{r}+H_{5}
$$

where $P_{r}$ and $T_{r}$ are reduced pressure of carbon dioxide $\left(P_{r}=\frac{P}{P_{c}}\right)$ and reduced temperature of carbon dioxide $\left(T_{r}=\frac{T}{T_{c}}\right)$, respectively. The $H_{1}-H_{5}$ are model constants. The $P_{c}$ and $T_{c}$ are critical pressure $\left(P_{c}=7.387 \mathrm{MPa}\right)$ and critical temperature $\left(T_{\mathcal{c}}=304.12 \mathrm{~K}\right)$, respectively.

\subsection{Solid-Liquid Equilibrium Criteria Model}

The behavior of solid solute in the liquid phase is determined by a ratio of the fugacity between pure liquid solute and the solid state at pressure $(P)$ and temperature $(T)$, which have reported elsewhere [4,16-18]. Moreover, the activity of substance obtained from the melting temperature and the melting enthalpy of compound. The activity coefficient of the substance can be represented by the regular solution model together with theory of Flory Huggins $[6,17,19]$. The solubility representation of the solute in $\mathrm{ScCO}_{2}$ is expressed by

$$
\ln y_{2}=\frac{\Delta H_{2}^{m}}{R T}\left(\frac{T}{T_{m}}-1\right)-\frac{v_{2}}{R T}\left(\delta_{1}-\delta_{2}\right)^{2}-\ln \left(\frac{v_{2}}{v_{1}}\right)-1+\frac{v_{2}}{v_{1}}
$$

where $\Delta H_{2}^{m}, T_{m}$, and $v_{2}$ are the enthalpy of melting, melting temperature, and molar volume of the solute, respectively. These data are presented in Table $1 . v_{1}$ is the molar volume of $\mathrm{ScCO}_{2} . \Delta H_{2}^{m}$ and $v_{2}$ are estimated by Jain et al. method [20] and by Fedors method [21], respectively. The solubility parameter of $\mathrm{ScCO}_{2}\left(\delta_{1}\right)$ is calculated by Giddings method [22],

$$
\delta_{1}=8.032\left(P_{c} / \mathrm{MPa}\right)^{0.5}\left(\frac{\rho_{r}}{2.66}\right)
$$

where $P_{c}$ is critical pressure $\left(P_{c}=7.387 \mathrm{MPa}\right), \rho_{r}$ is the reduced density of $\mathrm{CO}_{2}$, it can be calculated by $\rho_{r}=\frac{\rho}{\rho_{c}}$; the density of $\mathrm{ScCO}_{2}, \rho$, is obtained from website of NIST Web Book [23]. By assumption that the $\mathrm{ScCO}_{2}$ density depends on the solubility parameter of the solid solute $\left(\delta_{2}\right)$, the correlation can be expressed as:

$$
\delta_{2}=a+b \rho_{\mathrm{ScCO}_{2}}^{c}
$$

where $\rho_{\mathrm{ScCO}_{2}}$ is the density of $\mathrm{ScCO}_{2}$ in $\left(\mathrm{mol} / \mathrm{m}^{3}\right)$ obtained from website of NIST Web Book [23], $a, b$, and $c$ are adjustable parameters. 
Table 1. Physical properties of the compounds.

\begin{tabular}{|c|c|c|c|c|}
\hline \multicolumn{2}{|c|}{ Serial Number \& Name } & $T_{\mathrm{m}}$ & $\Delta H_{2}^{m} \mathrm{~b}(\mathrm{KJ} / \mathrm{mol})$ & $v_{2} \cdot 10^{4 \mathrm{c}}\left(\mathrm{m}^{3} / \mathrm{mol}\right)$ \\
\hline 1. & C.I. disperse blue 3 & $453.6^{\mathrm{a}}$ & 35.48 & 2.172 \\
\hline 2. & Blue 1 & $599.74^{b}$ & 34.42 & 1.894 \\
\hline 3. & 1,4-dihydroxy-9,10-anthraquinone & $469.15^{\mathrm{a}}$ & 27.81 & 1.665 \\
\hline 4. & $\begin{array}{l}\text { 1-Hydroxy-4-(prop-2-enyloxy)-9,10- } \\
\text { anthraquinone }\end{array}$ & $463.38^{b}$ & 31.74 & 2.030 \\
\hline 5. & $\begin{array}{l}\text { 1,4-bis(prop-2'-enyloxy)-9,10- } \\
\text { anthraquinone }\end{array}$ & $448.17^{b}$ & 35.67 & 2.280 \\
\hline 6. & 1-amino-2-methylanthraquinone & $478.15^{\mathrm{a}}$ & 24.12 & 1.789 \\
\hline 7. & 1- amino-2-ethyl-9,10-anthraquinone & $427.15^{a}$ & 26.95 & 1.732 \\
\hline 8. & $\begin{array}{l}\text { 1-amino-2,3- } \\
\text { dimethylanthraquinone }\end{array}$ & $486.15^{\mathrm{a}}$ & 24.60 & 1.790 \\
\hline 9. & 1-hydroxy-9,10-anthraquinone & $599.28^{a}$ & 23.92 & 1.610 \\
\hline 10. & 1-hydroxy-2-methylanthraquinone & $458.15^{\mathrm{a}}$ & 24.40 & 1.759 \\
\hline 11. & $\begin{array}{l}\text { 1-hydroxy-2-(methoxy } \\
\text { methyl)anthraquinone }\end{array}$ & $433.94^{b}$ & 29.72 & 1.964 \\
\hline 12. & $\begin{array}{l}\text { 1-hydroxyl-2-(ethoxy } \\
\text { methyl)anthraquinone }\end{array}$ & $401.15^{\mathrm{a}}$ & 32.55 & 2.113 \\
\hline 13. & $\begin{array}{l}\text { 1-hydroxy-2-(1-propoxy } \\
\text { methyl)anthraquinone }\end{array}$ & $424.0^{\mathrm{b}}$ & 35.39 & 2.263 \\
\hline 14. & $\begin{array}{l}\text { 1-hydroxy-2-(1-butoxymethyl) } \\
\text { anthraquinone }\end{array}$ & $389.74^{b}$ & 38.22 & 2.412 \\
\hline 15. & $\begin{array}{l}\text { 1-hydroxy-2-(n-amyloxy methyl) } \\
\text { anthraquinone }\end{array}$ & $418.64^{b}$ & 41.06 & 2.561 \\
\hline 16. & Quinizarin & $469.15^{\mathrm{a}}$ & 27.81 & 1.665 \\
\hline 17. & Violet 1(1,4-diaminoanthraquinone) & $539.15^{\mathrm{a}}$ & 27.23 & 1.178 \\
\hline 18. & $\begin{array}{l}\text { Blue } 59 \text { (1,4-bis (ethyl } \\
\text { amino)anthraquinone) }\end{array}$ & $471.15^{\mathrm{a}}$ & 33.04 & 1.880 \\
\hline 19. & $\begin{array}{l}\text { Red } 15 \\
\text { (1-amino-4-hydroxyanthraquinone) }\end{array}$ & $489.15^{\mathrm{a}}$ & 27.51 & 1.116 \\
\hline 20. & 1 hydroxy-4-nitroanthraquinone & $540^{\mathrm{a}}$ & 26.61 & 1.214 \\
\hline 21 & $\begin{array}{l}\text { 1,8-dihidroxy-4,5- } \\
\text { dinitroanthraquinone }\end{array}$ & $573.1^{\mathrm{a}}$ & 33.19 & 1.254 \\
\hline 22. & $\begin{array}{l}\text { 1,4 } \\
\text { diamino-2,3-dichloroanthraquinone }\end{array}$ & $576^{\mathrm{a}}$ & 29.38 & 1.758 \\
\hline 23. & 1-aminoanthraquinone & $526^{a}$ & 23.63 & 1.176 \\
\hline 24. & 1-nitroanthraquinone & $505.5^{\mathrm{a}}$ & 22.73 & 1.554 \\
\hline 25. & C.I. Disperse orange 11 & $478.15^{\mathrm{a}}$ & 24.12 & 1.789 \\
\hline
\end{tabular}

${ }^{a}$ From CAS databased. (https://scifinder.cas.org/scifinder/view/scifinder/scifinderExplore.jsf). ${ }^{b}$ Estimated by Jain et al. method [20]. ${ }^{c}$ Estimated by Fedors method [21].

\section{New Models}

\subsection{Model 1}

In solid-liquid equilibrium criteria, the supercritical phase is generally assumed as an expanded liquid consisting of infinite dissolved solute. At equilibrium, the solubility is expressed as $[2,13,14]$

$$
y_{2}=\frac{1}{\gamma_{2}^{\infty}} \frac{f_{2}^{S}}{f_{2}^{L}}
$$

In Equation (13), $\gamma_{2}^{\infty}$ is solute activity coefficient at infinite dilution in supercritical fluid and $f_{2}^{S}, f_{2}^{L}$ are fugacity of solute in solid phase and supercritical fluid phase, respectively. From thermodynamics, pure solid to pure liquid fugacity ratio is expressed [24] as

$$
\frac{f_{2}^{S}}{f_{2}^{L}}=\exp \left[\frac{\Delta H_{2}^{m}}{R T}\left(\frac{T}{T_{m}}-1\right)-\int_{T_{m}}^{T} \frac{1}{R T^{2}}\left[\int_{T_{m}}^{T}\left[\Delta C_{p}\right] d T\right] d T\right]
$$


In Equation (14), $\Delta C_{p}$ is known as difference in heat capacity between that of solid state minus liquid state, $R$ is well known as universal gas constant. Combining Equations (13) and (14) gives Equation (15) for solubility for a special case where $\Delta C_{p}$ is constant.

$$
y_{2}=\frac{1}{\gamma_{2}^{\infty}} \exp \left[\frac{\Delta H_{2}^{m}}{R T}\left(\frac{T}{T_{m}}-1\right)-\frac{\Delta C_{p}}{R}\left[\ln \left(\frac{T}{T_{m}}\right)-T_{m}\left(\frac{1}{T_{m}}-\frac{1}{T}\right)\right]\right]
$$

In Equation (15), the quantities $\Delta H_{2}^{m}$ and $T_{m}$ are constants for a given substance, therefore the exponential term in Equation (15) is written only in terms of temperature as

$$
y_{2}=\frac{1}{\gamma_{2}^{\infty}} \exp \left[a+\frac{b}{T}+c \ln (T)\right]
$$

In Equation (16), $N_{1}=\frac{\Delta H_{2}^{m}}{R T_{m}}+\frac{\Delta C_{p}}{R}\left[\ln \left(T_{m}\right)+1\right], N_{2}=\frac{-\Delta H_{2}^{m}}{R}-\frac{\Delta C_{p} T_{m}}{R}$ and $N_{3}=\frac{-\Delta C_{p}}{R}$.

The required activity coefficients in Equation (16) can be obtained from van Laar equation [24] as

$$
\ln \left(\gamma_{2}^{\infty}\right)=A_{21}\left[\frac{A_{12} y_{1}}{A_{12} y_{1}+A_{21} y_{2}}\right]^{2}
$$

Equation (17) combined with Equation (16) would give the new model as

$$
y_{2}=\exp \left[N_{1}+\frac{N_{2}}{T}+N_{3} \ln (T)\right] / \exp \left(A_{21}\left[\frac{A_{12} y_{1}}{A_{12} y_{1}+A_{21} y_{2}}\right]^{2}\right)
$$

Equation (18) represents the five parameter model derived based on solid and liquid phase equilibrium criterion and van Laar model for activity coefficient. In Equation (18), $N_{1}, N_{2}, N_{3}, A_{12}$ and $A_{21}$ are constants.

\subsection{Model 2}

In this model, the solid-liquid equilibrium criteria are the same as that of model 1. In place of pure solid to pure liquid fugacity ratio, a second order polynomial in temperature is considered [25]. The consideration may be justified from the actual expression for the fugacity ratio $[26,27]$, which is

$$
\ln \left(\frac{f_{s}}{f_{L}}\right)=\frac{\Delta H_{2}^{m}}{R}\left(\frac{1}{T}-\frac{1}{T_{m}}\right)-\frac{1}{R T} \int_{T_{m}}^{T} \Delta C_{p} d T+\frac{1}{R} \int_{T_{m}}^{T} \frac{\Delta C_{p}}{T} d T+\int_{P_{2}^{s a t}}^{P} \frac{v_{2}}{R T} d P
$$

Equation (19) gross form is a polynomial in temperature. The polynomial term (for temperature dependence) in literature is also observed with the work presented by Nordström and Rasmuson [25], who fitted the solubility of salicylamide in various solvents at normal pressures. Therefore, fugacity ratio in this work is expressed as a second order polynomial in terms of temperature as $\exp \left(A+B / T+C / T^{2}\right)$. Therefore, the final expression for solubility is

$$
y_{2}=\frac{1}{\gamma_{2}^{\infty}} \exp \left[A+\frac{B}{T}+\frac{C}{T^{2}}\right]
$$

The required activity coefficients in Equation (20) can be obtained from van Laar equation as in Equation (17). Equation (17) combined with Equation (20) would give the new model as

$$
y_{2}=\exp \left[A+\frac{B}{T}+\frac{C}{T^{2}}\right] / \exp \left(A_{21}\left[\frac{A_{12} y_{1}}{A_{12} y_{1}+A_{21} y_{2}}\right]^{2}\right)
$$


Equation (21) represents the five parameter model derived based on solid and liquid phase equilibrium criterion and van Laar model for activity coefficient. In Equation (21) $A$, $B, C, A_{12}$, and $A_{21}$ are constants.

\section{Methodology}

We used fminsearch algorithm which uses the Nelder-Mead simplex as described by Lagarias et al. [28] built in MATLAB software (R2019b) student version to fit models and experimental data collected from literature. Furthermore, we also inspected the quality of modeling through various entities such as correlation coefficient $\left(R^{2}\right)$, adjusted $R^{2}$ (Adj. $R^{2}$ ), root mean square deviation (RMSE), sum of squares due to error (SSE), and the overall average absolute relative deviation $(A A R D)$ between experimental data and calculated results. The $R^{2}$, Adj. $R^{2}, S S E$, and $R M S E$ are evaluated using the following formulas [29]

$$
\begin{gathered}
A A R D /(\%)=\frac{100}{\mathrm{~N}_{\mathrm{i}}} \sum_{\mathrm{i}=1}^{\mathrm{N}_{i}} \frac{\left|y_{2}^{\mathrm{cal}}-y_{2}^{\exp }\right|}{y_{2}^{\exp }} \\
R^{2}=1-\frac{\sum_{\mathrm{i}=1}^{\mathrm{N}_{\mathrm{i}}}\left(y_{2}^{\exp }-y_{2}^{\mathrm{cal}}\right)^{2}}{\sum_{\mathrm{i}=1}^{\mathrm{N}_{\mathrm{i}}}\left(y_{2}^{\overline{\exp }}-y_{2}^{\mathrm{cal}}\right)^{2}} \\
\text { Adj. } R^{2}=R^{2}-\frac{Q\left(1-R^{2}\right)}{N_{i}-Q-1} \\
\text { SSE }=\left[\sum_{i=1}^{\mathrm{N}_{\mathrm{i}}}\left(y_{2}^{\exp }-y_{2}^{\mathrm{cal}}\right)^{2}\right] \\
\text { RMSE }=\left[\frac{1}{N_{i}} \sum_{i=1}^{\mathrm{N}_{\mathrm{i}}}\left(y_{2}^{\exp }-y_{2}^{\mathrm{cal}}\right)^{2}\right]^{\frac{1}{2}}
\end{gathered}
$$

In Equation (22), $y_{2}^{\mathrm{cal}}$ and $y_{2}^{\exp }$ represent the mole fraction of calculated and experimental solubility's values, respectively. $y_{2}^{\overline{\exp }}$ is the global mean value of experimental data in mole fraction.

Statistical comparison of models is essential to ensure the success of the new model. In order to this achieve this, the well-known Akaike's Information Criterion (AIC) proposed by Akaike $[30,31]$ has been used. AIC is expressed as

$$
\mathrm{AIC}=N \ln \left(\frac{S S E}{N}\right)+2 K
$$

In Equation (27), $K$ is number of parameter constants, $N$ is number of data points, $S S E$ is the sum of squares due to error. Importantly, AIC is number of adjustable parameters of the individual model.

\section{Results and Discussion}

In this study, we propose two new solid-liquid equilibrium criteria models to correlate solubility of solid in supercritical carbon dioxide. The accuracy of the proposed models is evaluated by correlating 25 anthraquinone derivative compounds available in the literature. The correlating ability of the new models are evaluated in terms of: $A A R D, R^{2}, \operatorname{Adj} . R^{2}$, $S S E$, and RMSE. There are more than 25 models available in literature [29] for correlating solubility of solids in supercritical fluids. However, for comparison purposes, we have considered Chrastil model, Adachi and Lu model, Mitra-Wilson model, Keshmiri et al. model, Khansary et al. model, Garlapati and Madras model, Reddy et al., model, and one existing three parameters solid-liquid equilibrium model. These are grouped as three parameter models and five parameter models. Table 2 shows the information of the 25 
anthraquinone derivatives considered in this study. Table 2 shows the solubility range and references $[4,16-18,32-38]$ from which the data are obtained. Table 1 shows the physical properties such as melting point, melting enthalpy, and molar volume of the solutes. For some compounds, these properties are not available and for such compounds we have used the Jain et al. method [20] and Fedors method [21] for evaluating the melting enthalpy and solute molar volumes, respectively. The constant parameters of literature models considered, Chrastil, Adachi-Lu, Mitra-Wilson, Keshmiri et al., Khansary et al., Bian et al., GarlapatiMadras, and Reddy et al., are listed in the Supplementary Materials (Tables S1-S8). Table 3 shows the correlation results of the three parameter solid-liquid equilibrium model. Table 4 shows the correlation constants of the new model 1 . Table 5 shows the correlation constants of the new model 2. Table 6 shows the overall mean statistical parameters of various solubility models. From Table 6, it is clear that the proposed models show the lowest AARD. The new model 1 shows an overall AARD\% of 6.538 and the second model (new model 2) shows an overall AARD\% of 6.377. The two models proposed in this work are observed to perform the correlation on a par. Although they look different in functional form, their correlation ability is matching well. This correlating matching ability may be attributed to its oneness in their functional form.

Table 2. Solubility information of the compounds.

\begin{tabular}{|c|c|c|c|c|c|c|}
\hline \multicolumn{2}{|c|}{ Serial Number and Name } & \multirow[t]{2}{*}{ Chemical Structure } & \multirow{2}{*}{$\begin{array}{c}\begin{array}{c}\text { Solubility } \\
\text { Range } y_{2} \times 10^{6}\end{array} \\
0.68-63.575\end{array}$} & \multirow{2}{*}{$\begin{array}{c}\mathbf{T}(\mathbf{K}) \text { and } \\
\text { P(MPa) Range } \\
\\
(323.7-413.7) ; \\
(10.51-32.98)\end{array}$} & \multirow{2}{*}{$\begin{array}{l}\mathbf{N} \\
23\end{array}$} & \multirow{2}{*}{$\begin{array}{c}\text { Reference } \\
\text { [33] }\end{array}$} \\
\hline 1. & C.I. disperse blue 3 & & & & & \\
\hline 2. & Blue 1 & & $6.63-44.5$ & $\begin{array}{c}(333.3-373.2) ; \\
(20-40)\end{array}$ & 18 & [34] \\
\hline 3. & $\begin{array}{l}\text { 1,4-dihydroxy-9,10- } \\
\text { anthraquinone }\end{array}$ & & $13-314$ & $\begin{array}{c}(308-348) ; \\
(12.16-40.53)\end{array}$ & 40 & {$[35,36]$} \\
\hline 4. & $\begin{array}{l}\text { 1-Hydroxy-4-(prop- } \\
2^{\prime} \text {-enyloxy)-9,10- } \\
\text { anthraquinone }\end{array}$ & & 9-498 & $\begin{array}{c}(308-348) ; \\
(12.16-40.53)\end{array}$ & 38 & [36] \\
\hline 5. & $\begin{array}{l}\text { 1,4-bis(prop-2'- } \\
\text { enyloxy)-9,10- } \\
\text { anthraquinone }\end{array}$ & & $2-200$ & $\begin{array}{c}(308-348) ; \\
(12.16-40.53)\end{array}$ & 34 & [36] \\
\hline 6. & $\begin{array}{l}\text { 1-amino-2- } \\
\text { methylanthraquinone }\end{array}$ & & $4.6-109.6$ & $\begin{array}{l}(308-348) ; \\
(12.2-35.5)\end{array}$ & 43 & [37] \\
\hline
\end{tabular}


Table 2. Cont.

\begin{tabular}{|c|c|c|c|c|c|}
\hline 7. & $\begin{array}{l}\text { 1- amino-2-ethyl-9,10- } \\
\text { anthraquinone }\end{array}$ & $2.6-77.8$ & $\begin{array}{l}(308-348) \\
(12.2-35.5)\end{array}$ & 43 & [37] \\
\hline 8. & $\begin{array}{l}\text { 1-amino-2,3- } \\
\text { dimethylanthraquinone }\end{array}$ & $4.6-37.9$ & $\begin{array}{l}(308-348) \\
(12.2-35.5)\end{array}$ & 41 & [37] \\
\hline 9. & $\begin{array}{l}\text { 1-hydroxy-9,10- } \\
\text { anthraquinone }\end{array}$ & $30-445$ & $\begin{array}{l}(308-348) \\
(12.2-35.5)\end{array}$ & 45 & [38] \\
\hline 10. & $\begin{array}{l}\text { 1-hydroxy-2- } \\
\text { methylanthraquinone }\end{array}$ & $9-737$ & $\begin{array}{l}(308-348) ; \\
(12.2-35.5)\end{array}$ & 45 & [38] \\
\hline 11. & $\begin{array}{l}\text { 1-hydroxy-2- } \\
\text { (methoxy } \\
\text { methyl)anthraquinone }\end{array}$ & $1-537$ & $\begin{array}{l}(308-348) ; \\
(12.2-35.5)\end{array}$ & 45 & [38] \\
\hline 12. & $\begin{array}{l}\text { 1-hydroxyl-2-(ethoxy } \\
\text { methyl)anthraquinone }\end{array}$ & $23-1100$ & $\begin{array}{l}(308-348) \\
(12.2-35.5)\end{array}$ & 45 & [38] \\
\hline 13. & $\begin{array}{l}\text { 1-hydroxy-2-(1- } \\
\text { propoxy } \\
\text { methyl)anthraquinone }\end{array}$ & 103-1676 & $\begin{array}{l}(308-348) ; \\
(12.2-35.5)\end{array}$ & 45 & [38] \\
\hline 14. & $\begin{array}{l}\text { 1-hydroxy-2-(1- } \\
\text { butoxymethyl) } \\
\text { anthraquinone }\end{array}$ & $82-2699$ & $\begin{array}{l}(308-348) ; \\
(12.2-35.5)\end{array}$ & 45 & [38] \\
\hline 15. & $\begin{array}{l}\text { 1-hydroxy-2-(n- } \\
\text { amyloxy methyl) } \\
\text { anthraquinone }\end{array}$ & $38-2640$ & $\begin{array}{r}(308-348) ; \\
(12.2-35.5)\end{array}$ & 45 & [38] \\
\hline 16. & Quinizarin & $69-6940$ & $\begin{array}{l}(353.2-393.2) \\
\quad(12-30)\end{array}$ & 15 & {$[35,36]$} \\
\hline 17. & $\begin{array}{l}\text { Violet } 1(1,4- \\
\text { diaminoanthraquinone) }\end{array}$ & $0.13-2.61$ & $\begin{array}{c}(323.15-383.15) \\
(15-25)\end{array}$ & 15 & [16] \\
\hline
\end{tabular}


Table 2. Cont.

\begin{tabular}{|c|c|c|c|c|c|}
\hline 18. & $\begin{array}{l}\text { Blue } 59 \text { (1,4-bis (ethyl } \\
\text { amino)anthraquinone) }\end{array}$ & $0.218-14.9$ & $\begin{array}{c}(323.15-383.15) \\
\quad(12.5-25)\end{array}$ & 26 & [16] \\
\hline 19. & $\begin{array}{l}\text { Red } 15 \text { (1-amino-4- } \\
\text { hydroxyanthraquinone) }\end{array}$ & $1.84-24.5$ & $\begin{array}{c}(323.15-383.15) \\
\quad(12.5-25)\end{array}$ & 20 & [18] \\
\hline 20. & $\begin{array}{l}1 \text { hydroxy-4- } \\
\text { nitroanthraquinone }\end{array}$ & $1.22-8.64$ & $\begin{array}{c}(323.15-383.15) \\
(15-25)\end{array}$ & 15 & [18] \\
\hline 21 & $\begin{array}{l}\text { 1,8-dihidroxy-4,5- } \\
\text { dinitroanthraquinone }\end{array}$ & 0.168-1.12 & $\begin{array}{c}(323.15-383.15) \\
(15-25)\end{array}$ & 15 & [4] \\
\hline 22. & $\begin{array}{l}\text { 1,4 diamino-2,3- } \\
\text { dichloroanthraquinone }\end{array}$ & $0.053-5.24$ & $\begin{array}{c}(323.15-383.15) \\
(12.5-25)\end{array}$ & 18 & [4] \\
\hline 23. & $\begin{array}{l}\text { 1- } \\
\text { aminoanthraquinone }\end{array}$ & $0.55-35.1$ & $\begin{array}{c}(323.15-383.15) \\
\quad(12.5-25)\end{array}$ & 18 & [17] \\
\hline 24. & $\begin{array}{l}\text { 1- } \\
\text { nitroanthraquinone }\end{array}$ & $0.984-25.2$ & $\begin{array}{c}(323.15-383.15) \\
\quad(12.5-25)\end{array}$ & 18 & [17] \\
\hline 25. & $\begin{array}{l}\text { C.I. Disperse orange } \\
11\end{array}$ & $0.58-30.3$ & $\begin{array}{c}(323.15-383.15) \\
(12-25)\end{array}$ & 12 & [32] \\
\hline
\end{tabular}


Table 3. Correlation results of the three parameter solid-liquid equilibrium model (Equations (10)-(12)).

\begin{tabular}{ccccc}
\hline S1.No* & $\boldsymbol{a}$ & $\boldsymbol{b}$ & $\boldsymbol{c}$ & AARD $\%$ \\
\hline 1 & 16,983 & 0.111170 & 1.15140 & 55.328 \\
2 & 14,423 & 8.083700 & 0.76039 & 34.399 \\
3 & 14,867 & 0.524320 & 1.01780 & 12.935 \\
4 & 13,786 & 0.390040 & 1.04700 & 14.982 \\
5 & 15,545 & 0.025248 & 1.30360 & 36.224 \\
6 & 14,282 & 1.375900 & 0.92731 & 16.165 \\
7 & 17,441 & 0.022117 & 1.31830 & 25.785 \\
8 & 19,195 & 0.002538 & 1.52380 & 23.006 \\
9 & 13,508 & 0.477910 & 1.03180 & 12.740 \\
10 & 16,683 & 0.021064 & 1.32190 & 8.198 \\
11 & 15,754 & 0.000044 & 1.97910 & 82.871 \\
12 & 13,395 & 0.693360 & 0.99182 & 18.228 \\
13 & 13,574 & 0.153700 & 1.13460 & 16.541 \\
14 & 10,512 & 2.867000 & 0.86305 & 17.309 \\
15 & 13,936 & 0.081591 & 1.18840 & 36.363 \\
16 & 16,800 & 0.000017 & 2.01370 & 32.140 \\
17 & 22,206 & 0.000122 & 1.59150 & 39.940 \\
18 & 18,628 & 0.010357 & 1.38690 & 8.268 \\
19 & 22,846 & 0.009591 & 1.38010 & 6.304 \\
20 & 22,936 & 0.001514 & 1.56360 & 11.761 \\
21 & 23,417 & 0.001591 & 1.55150 & 28.828 \\
22 & 19,181 & 0.005504 & 1.45030 & 14.566 \\
23 & 22,218 & 0.002795 & 1.50580 & 5.625 \\
24 & 18,613 & 0.096199 & 1.17250 & 10.777 \\
25 & 18,221 & 0.010624 & 1.39170 & 20.698 \\
\hline
\end{tabular}

Sl.No*: Serial number and name same as Table 2.

Table 4. Correlation constants of the new model 1 (Equation (18)).

\begin{tabular}{|c|c|c|c|c|c|c|c|c|c|c|}
\hline S1.No* & $A_{12}$ & $A_{21} \cdot 10^{5}$ & $N_{1}$ & $N_{2}$ & $N_{3}$ & AARD $\%$ & $R^{2}$ & $\operatorname{Adj} . R^{2}$ & $R M S E \cdot 10^{7}$ & $S S E \cdot 10^{16}$ \\
\hline 1 & 4.6317 & 7.4408 & -123.220 & 5849.4 & 16.501 & 10.3260 & 0.925 & 0.904 & 30.93 & $2,295,600$ \\
\hline 2 & 3.6237 & 0.1369 & -22.280 & 491.4 & 1.197 & 1.2124 & 0.998 & 0.997 & 0.04 & 2.85400 \\
\hline 3 & 3.8856 & 56.018 & -119.110 & 5355.7 & 16.390 & 4.3482 & 0.980 & 0.977 & 76.09 & $23,159,000$ \\
\hline 4 & 4.1249 & 66.839 & -255.500 & 11861.0 & 36.544 & 7.8210 & 0.802 & 0.773 & 185.89 & $138,230,000$ \\
\hline 5 & 4.4232 & 34.492 & 352.360 & -17338.0 & -53.119 & 7.8357 & 0.905 & 0.891 & 96.46 & $37,222,000$ \\
\hline 6 & 3.9076 & 20.069 & -35.941 & 1222.0 & 4.032 & 4.6621 & 0.949 & 0.943 & 28.25 & $3,592,400$ \\
\hline 7 & 4.3202 & 11.976 & -44.086 & 1418.5 & 5.251 & 5.8040 & 0.941 & 9.340 & 29.90 & $4,023,100$ \\
\hline 8 & 3.7536 & 10.665 & -133.510 & 5959.7 & 18.268 & 2.3299 & 0.981 & 0.979 & 6.39 & 183,520 \\
\hline 9 & 3.7520 & 100.30 & -65.421 & 2831.4 & 8.549 & 2.5184 & 0.960 & 0.954 & 66.34 & $19,807,000$ \\
\hline 10 & 4.1139 & 100.84 & -118.880 & 5233.1 & 16.522 & 8.1326 & 0.945 & 0.938 & 308.08 & $427,110,000$ \\
\hline 11 & 3.6917 & 132.15 & -81.270 & 3572.1 & 10.940 & 8.1276 & 0.843 & 0.823 & 309.64 & $431,440,000$ \\
\hline 12 & 3.8667 & 183.28 & -54.582 & 2282.9 & 7.074 & 4.9246 & 0.960 & 0.955 & 300.20 & $405,550,000$ \\
\hline 13 & 3.8025 & 327.28 & -73.225 & 3236.9 & 9.889 & 3.6837 & 0.963 & 0.958 & 333.17 & $499,500,000$ \\
\hline 14 & 4.0108 & 399.95 & -203.750 & 9484.9 & 29.170 & 6.1019 & 0.964 & 0.960 & 825.19 & $3,064,200,000$ \\
\hline 15 & 4.2943 & 294.42 & -80.585 & 3362.6 & 11.087 & 9.7765 & 0.919 & 0.909 & 1267.30 & $7,226,900,000$ \\
\hline 16 & 4.8875 & 1186.7 & 967.570 & -52133.0 & -140.540 & 11.4640 & 0.906 & 0.867 & 8137.90 & $119,200,000,000$ \\
\hline 17 & 3.8754 & 0.4939 & -95.533 & 4234.2 & 12.097 & 4.6045 & 0.946 & 0.924 & 0.66 & 657.72 \\
\hline 18 & 4.2476 & 2.0376 & -114.860 & 5180.0 & 15.189 & 10.5440 & 0.967 & 0.960 & 6.82 & 139,560 \\
\hline 19 & 4.1111 & 3.8448 & -141.350 & 6609.0 & 19.113 & 8.9540 & 0.922 & 0.879 & 9.75 & 199,440 \\
\hline 20 & 3.7799 & 1.8628 & -78.114 & 3418.2 & 9.746 & 3.1992 & 0.993 & 0.989 & 1.63 & 3990.60 \\
\hline 21 & 3.5828 & 0.3210 & -31.261 & 922.3 & 2.662 & 1.1563 & 0.973 & 0.958 & 0.10 & 13.78 \\
\hline 22 & 4.3364 & 0.5898 & -148.990 & 6728.0 & 20.046 & 10.9930 & 0.891 & 0.831 & 2.82 & 14339 \\
\hline 23 & 4.1502 & 4.8759 & -128.930 & 5935.5 & 17.365 & 9.4322 & 0.903 & 0.848 & 14.82 & 395,570 \\
\hline 24 & 3.9199 & 4.3919 & -67.609 & 2866.9 & 8.373 & 5.3605 & 0.977 & 0.965 & 7.33 & 96,592 \\
\hline 25 & 4.3723 & 3.6415 & 28.875 & -2317.9 & -5.576 & 10.1480 & 0.970 & 0.953 & 16.25 & 316,930 \\
\hline
\end{tabular}


Table 5. Correlation constants of the new model 2 (Equation (21)).

\begin{tabular}{|c|c|c|c|c|c|c|c|c|c|c|}
\hline S1.No* & $A_{12}$ & $A_{21} \cdot 10^{5}$ & $A$ & $B$ & $C \cdot 10^{-5}$ & AARD $/ \%$ & $R^{2}$ & $\operatorname{Adj} . R^{2}$ & $R M S E \cdot 10^{12}$ & $S S E \cdot 10^{6}$ \\
\hline 1 & 4.6319 & 7.461 & -1.0778 & -6194.3 & 10.941 & 10.347 & 0.925 & 0.904 & 229.99000 & 3.0956 \\
\hline 2 & 3.6237 & 0.137 & -13.461 & -353.46 & 0.744 & 1.2124 & 1.000 & 1.000 & 0.00029 & 0.0040 \\
\hline 3 & 3.8855 & 56.020 & 0.35304 & -5347.8 & 8.728 & 4.3504 & 0.980 & 0.977 & 2319.70 & 7.6153 \\
\hline 4 & 4.1251 & 66.837 & 10.906 & -12028 & 19.499 & 7.8214 & 0.947 & 0.940 & 13855.00 & 18.6110 \\
\hline 5 & 4.4208 & 34.554 & -34.627 & 17225 & -28.081 & 7.8227 & 0.905 & 0.891 & 3719.90 & 9.6436 \\
\hline 6 & 3.9077 & 20.070 & -6.642 & -1350.7 & 2.048 & 4.6627 & 0.949 & 0.943 & 360.00 & 2.8284 \\
\hline 7 & 4.3203 & 11.977 & -5.9057 & -1950 & 2.697 & 5.8043 & 0.941 & 0.934 & 403.16 & 2.9932 \\
\hline 8 & 3.7542 & 10.660 & -0.2361 & -6047.9 & 9.856 & 2.3265 & 0.981 & 0.979 & 18.39 & 0.6393 \\
\hline 9 & 3.752 & 100.310 & -3.0674 & -2776.4 & 4.593 & 2.5168 & 0.961 & 0.956 & 1983.30 & 6.6387 \\
\hline 10 & 4.114 & 100.850 & 1.5165 & -5534 & 8.762 & 8.1321 & 0.945 & 0.938 & $42,770.00$ & 30.8290 \\
\hline 11 & 3.7153 & 120.250 & 0.29924 & -4765.9 & 7.677 & 4.0867 & 0.984 & 0.982 & 2753.40 & 7.8222 \\
\hline 12 & 3.8668 & 183.240 & -2.9085 & -2409.6 & 3.886 & 4.9225 & 0.960 & 0.954 & 40,519 & 30.0070 \\
\hline 13 & 3.8026 & 327.230 & -1.0388 & -3291.2 & 5.381 & 3.6796 & 0.963 & 0.958 & 49,885 & 33.2950 \\
\hline 14 & 4.0108 & 400.240 & 8.7869 & -9511.2 & 15.448 & 6.1073 & 0.965 & 0.960 & 307,640 & 82.6830 \\
\hline 15 & 4.2944 & 294.450 & -0.13121 & -3641.3 & 5.518 & 9.7817 & 0.919 & 0.909 & 725,400 & 126.960 \\
\hline 16 & 4.8875 & 1186.800 & -75.478 & 52,674 & -97.606 & 11.464 & 0.906 & 0.867 & $11,919,000$ & 813.740 \\
\hline 17 & 3.8754 & 0.494 & -6.4227 & -4295.6 & 7.500 & 4.6045 & 0.946 & 0.916 & 0.06577 & 0.0662 \\
\hline 18 & 4.2476 & 2.038 & -2.9703 & -5529.4 & 9.416 & 10.544 & 0.967 & 0.960 & 13.9560 & 0.6820 \\
\hline 19 & 4.1111 & 3.845 & -0.55598 & -6866.1 & 11.846 & 8.954 & 0.922 & 0.896 & 19.9440 & 0.9745 \\
\hline 20 & 3.7799 & 1.863 & -6.3245 & -3452.8 & 6.041 & 3.1992 & 0.993 & 0.989 & 0.39906 & 0.1631 \\
\hline 21 & 3.5828 & 0.321 & -11.654 & -954.12 & 1.649 & 1.1563 & 0.973 & 0.958 & 0.00138 & 0.0096 \\
\hline 22 & 4.3364 & 0.590 & -1.3261 & -7404.6 & 12.425 & 10.993 & 0.891 & 0.846 & 1.43390 & 0.2823 \\
\hline 23 & 4.1502 & 4.876 & -1.0106 & -6311.3 & 10.770 & 9.4324 & 0.903 & 0.862 & 39.5560 & 1.4824 \\
\hline 24 & 3.9199 & 4.392 & -5.9336 & -3036 & 5.190 & 5.3605 & 0.977 & 0.968 & 9.65920 & 0.7326 \\
\hline 25 & 4.3723 & 3.642 & -12.201 & 1613.4 & -3.456 & 10.148 & 0.970 & 0.945 & 31.6930 & 1.6251 \\
\hline
\end{tabular}

Sl.No*: Serial number and name same as Table 2.

Table 6. Overall mean statistical parameters of solubility models.

\begin{tabular}{lcccccc}
\hline Model & $\begin{array}{c}\text { No. of } \\
\text { Constants }\end{array}$ & $\boldsymbol{R}^{\mathbf{2}}$ & Adj. $\boldsymbol{R}^{\mathbf{2}}$ & SSE & RMSE & AARD \% \\
\hline $\begin{array}{l}\text { Chrastil } \\
\text { Adachi-Lu }\end{array}$ & 3 & 0.89690 & 0.89295 & $1.30 \times 10^{-6}$ & $1.01 \times 10^{-4}$ & 17.485 \\
$\begin{array}{l}\text { Mitra- } \\
\text { Wilson }\end{array}$ & 5 & 0.89850 & 0.89780 & 1.27 & $7.69 \times 10^{-2}$ & 15.130 \\
$\begin{array}{l}\text { Keshmiri } \\
\text { et al. }\end{array}$ & 5 & 0.87990 & 0.87560 & 3.539 & $1.70 \times 10^{-1}$ & 21.240 \\
$\begin{array}{l}\text { Khansary } \\
\text { et al. }\end{array}$ & 5 & 0.89100 & 0.88700 & $6.67 \times 10^{-7}$ & $6.24 \times 10^{-5}$ & 17.530 \\
$\begin{array}{l}\text { Bian et al. } \\
\text { Garlapati- }\end{array}$ & 5 & 0.89644 & 0.89278 & $2.69 \times 10^{-7}$ & $4.97 \times 10^{-5}$ & 18.251 \\
$\begin{array}{l}\text { Madras } \\
\text { Reddy }\end{array}$ & 5 & 0.87800 & 0.87300 & $2.01 \times 10^{-7}$ & $4.53 \times 10^{-5}$ & 16.599 \\
$\begin{array}{l}\text { et al. } \\
\text { SLE model }\end{array}$ & 5 & 0.75600 & 0.74400 & $3.92 \times 10^{-6}$ & $1.50 \times 10^{-4}$ & 29.711 \\
$\begin{array}{l}\text { New } \\
\text { Model 1 }\end{array}$ & 5 & 0.88985 & 0.86064 & $4.44 \times 10^{-6}$ & $1.77 \times 10^{-4}$ & 23.599 \\
$\begin{array}{l}\text { New } \\
\text { Model 2 }\end{array}$ & 5 & 0.93930 & 0.92270 & $5.26 \times 10^{-7}$ & $4.825 \times 10^{-5}$ & 6.538 \\
\hline
\end{tabular}

To know the efficacy of the proposed models, further analysis is carried out with paired t-test and Akaike's Information Criterion (AIC). Table 7 shows the paired t-test (paired t-test, $p<0.05$ ) results for AARD, $R^{2}$, and Adj. $R^{2}$. From the results, it is clear that AARDs of the new models are statistically significant. Table 7 shows the paired t-test results for SSE and RMSE. From the results, it is clear that SSEs of the new models are not statistically significant. $R^{2}$, Adj. $R^{2}$, and $R M S E$ are showing mixed results and hence we 
could not infer any statistical meaning from them such as significant or not significant. Table 8 shows AIC information of the proposed models and literature models. From Tables 4 and 5 , the new models are significantly different at $95 \%$ confidence level (paired t-test, $p<0.05$ ). Table 8 of AIC information shows that among all models, the new models are having lower AIC values. The AIC value for the new model 1 is -730.59 , and for the new model 2 is -1177.56 . The lower AIC value indicates the goodness of the new models and we conclude that those models are superior to other models considered in the work.

Table 7. a. Paired $t$-test results for averaged absolute relative deviation (AARD), $R^{2}$ and Adj. $R^{2}, \mathrm{~b}$. Paired $t$-test results for sum of squares due to error (SSE) and RMSE.

\begin{tabular}{|c|c|c|c|c|c|c|}
\hline \multicolumn{7}{|c|}{ Paired $t$-test Results for AARD, $R^{2}$ and Adj. $R^{2}$} \\
\hline \multirow[b]{2}{*}{ Models } & \multicolumn{2}{|c|}{ AARD } & \multicolumn{2}{|c|}{$R^{2}$} & \multicolumn{2}{|c|}{ Adj. $R^{2}$} \\
\hline & $\begin{array}{c}\text { New } \\
\text { Model } 1\end{array}$ & $\begin{array}{c}\text { New } \\
\text { Model } 2\end{array}$ & $\begin{array}{c}\text { New } \\
\text { Model } 1\end{array}$ & $\begin{array}{c}\text { New } \\
\text { Model } 2\end{array}$ & $\begin{array}{c}\text { New } \\
\text { Model } 1\end{array}$ & $\begin{array}{c}\text { New } \\
\text { Model } 2\end{array}$ \\
\hline Chrastil & $S$ & $S$ & NS & NS & NS & NS \\
\hline Adachi-Lu & $S$ & $S$ & NS & NS & NS & NS \\
\hline Mitra-Wilson & $S$ & $S$ & $S$ & $\mathrm{~S}$ & NS & NS \\
\hline Keshmiri et al. & $S$ & S & NS & NS & NS & NS \\
\hline Khansary et al. & $S$ & $S$ & $S$ & $S$ & $S$ & NS \\
\hline Bian et al. & $S$ & $S$ & NS & NS & NS & NS \\
\hline $\begin{array}{l}\text { Garlapati- } \\
\text { Madras }\end{array}$ & S & S & $\mathrm{S}$ & S & NS & NS \\
\hline Reddy et al. & S & $S$ & S & S & NS & S \\
\hline SLE model & $S$ & $S$ & $S$ & $S$ & NS & NS \\
\hline \multicolumn{7}{|c|}{ Paired $t$-test Results for SSE and RMSE } \\
\hline \multirow[b]{2}{*}{ Models } & \multicolumn{3}{|c|}{ SSE } & \multicolumn{3}{|c|}{ RMSE } \\
\hline & New & odel 1 & $\begin{array}{c}\text { New } \\
\text { Model } 2\end{array}$ & Nen & odel 1 & $\begin{array}{c}\text { New } \\
\text { Model } 2\end{array}$ \\
\hline Chrastil & \multicolumn{2}{|c|}{ NS } & NS & \multicolumn{2}{|c|}{ NS } & NS \\
\hline Adachi-Lu & \multicolumn{2}{|c|}{ NS } & NS & \multicolumn{2}{|c|}{$S$} & NS \\
\hline Mitra-Wilson & \multicolumn{2}{|c|}{ NS } & NS & \multicolumn{2}{|c|}{$\mathrm{S}$} & S \\
\hline Keshmiri et al. & \multicolumn{2}{|c|}{ NS } & NS & \multicolumn{2}{|c|}{ NS } & NS \\
\hline Khansary et al. & \multicolumn{2}{|c|}{ NS } & NS & \multicolumn{2}{|c|}{$S$} & NS \\
\hline Bian et al. & \multicolumn{2}{|c|}{ NS } & NS & \multicolumn{2}{|c|}{ NS } & NS \\
\hline $\begin{array}{l}\text { Garlapati- } \\
\text { Madras }\end{array}$ & \multicolumn{2}{|c|}{ NS } & NS & \multicolumn{2}{|c|}{ NS } & NS \\
\hline Reddy et al. & \multirow{2}{*}{\multicolumn{2}{|c|}{$\begin{array}{l}\text { NS } \\
\text { NS }\end{array}$}} & NS & \multicolumn{2}{|c|}{ NS } & NS \\
\hline SLE model & & & NS & \multicolumn{2}{|c|}{ NS } & NS \\
\hline
\end{tabular}

NS: Not significant; S: Significant. 
Table 8. AIC information of the proposed models and literature models.

\begin{tabular}{|c|c|c|c|c|c|c|c|c|c|c|c|}
\hline Sl.No* & $\begin{array}{c}\text { Equation } \\
\text { (18) }\end{array}$ & $\begin{array}{l}\text { Equation } \\
\text { (21) }\end{array}$ & $\begin{array}{c}\text { Equation } \\
\text { (2) }\end{array}$ & $\begin{array}{l}\text { Equation } \\
\text { (3) }\end{array}$ & $\begin{array}{l}\text { Equation } \\
\text { (4) }\end{array}$ & $\begin{array}{c}\text { Equation } \\
\text { (5) }\end{array}$ & $\begin{array}{c}\text { Equation } \\
\text { (6) }\end{array}$ & $\begin{array}{c}\text { Equation } \\
\text { (7) }\end{array}$ & $\begin{array}{c}\text { Equation } \\
\text { (8) }\end{array}$ & $\begin{array}{l}\text { Equation } \\
\text { (9) }\end{array}$ & $\begin{array}{l}\text { SLE } \\
\text { (Equa- } \\
\text { tions } \\
(\mathbf{1 0 )}-(\mathbf{1 2}))\end{array}$ \\
\hline 1 & 72.60 & -883.48 & -543.41 & -547.95 & -544.26 & -529.96 & -533.91 & -548.37 & -521.14 & -520.16 & -502.02916 \\
\hline 2 & 686.29 & -804.56 & -604.41 & -296.42 & -285.04 & -603.40 & -608.51 & -587.68 & -602.21 & -583.75 & -577.05251 \\
\hline 3 & -932.89 & -1530.00 & -968.16 & -303.79 & -230.96 & -975.80 & -948.67 & -929.28 & -880.29 & -824.08 & -904.08437 \\
\hline 4 & -815.91 & -1417.10 & -711.57 & -53.04 & -44.02 & -700.99 & -706.31 & -693.52 & -698 & -67 & -692.25585 \\
\hline 5 & -769.80 & -1285.45 & -730.70 & -140.86 & -99.47 & -731 & -724.22 & -704.18 & -716.47 & -677.58 & -656.16322 \\
\hline 6 & -1086.85 & -1691.20 & -1051.84 & -326.98 & -284.62 & -1041.34 & -1035.41 & -1010.52 & -1017.78 & -951.70 & -999.79284 \\
\hline 7 & -1081.98 & -1688.77 & -1001.05 & -257.98 & -232.93 & -1006.04 & -1001.16 & -1009.39 & -999.11 & -945.54 & -960.43326 \\
\hline 8 & -1155.83 & -1671.09 & -1022.17 & -331.42 & -313.68 & -1020.25 & -1035.60 & -1005.96 & -977.98 & -980 & -1008.7995 \\
\hline 9 & -1063.09 & -1733.98 & -973.50 & -220.08 & -210.03 & -954.59 & -955.82 & -973.06 & -975.24 & -894.61 & -974.00663 \\
\hline 10 & -924.90 & -1664.88 & -970.35 & -215.20 & -156.93 & -961.05 & -951.72 & -929.51 & -928.08 & -832.69 & -975.57241 \\
\hline 11 & -924.44 & -1726.60 & -815.67 & -760.86 & -930.34 & -820.67 & -943.42 & -811.84 & -808.04 & -986.59 & -921.64403 \\
\hline 12 & -927.23 & -1666.10 & -913.68 & -153.10 & -143.13 & -916.41 & -889.74 & -923.98 & -887.23 & -849.04 & -900.93092 \\
\hline 13 & -917.85 & -1661.42 & -876.78 & -128.34 & -80.13 & -883.27 & -875.88 & -866.40 & -821.62 & -790.88 & -871.09925 \\
\hline 14 & -836.22 & -1620.49 & -815.23 & -26.09 & -22.74 & -795.27 & -789.52 & -795.24 & -845.10 & -743.41 & -815.42235 \\
\hline 15 & -797.61 & -1601.19 & -790.56 & -791.01 & -764.98 & -787.64 & -774.85 & -789.17 & -793.49 & -732.22 & -773.78737 \\
\hline 16 & -200.68 & -482.72 & -218.20 & -189.25 & 25.92 & -216.10 & -196.21 & -217.34 & -223.00 & -170.32 & -202.70016 \\
\hline 17 & -485.91 & -623.97 & -270.41 & -234.37 & -238.65 & -486.03 & -469.66 & -494.58 & -491.75 & -463.79 & -173.59318 \\
\hline 18 & -724.58 & -1042.54 & -758.18 & -324 & -304.19 & -729 & -732.67 & -755.40 & -699.79 & -705.83 & -763.27385 \\
\hline 19 & -542.68 & -787.26 & -265.77 & -241.05 & -228.48 & -547.15 & -532.15 & -545.48 & -514.00 & -533.35 & -569.89688 \\
\hline 20 & -458.87 & -610.44 & -438.25 & -182.81 & -199.53 & -429.90 & -419.25 & -456.83 & -432.72 & -433.09 & -441.6821 \\
\hline 21 & -543.89 & -652.93 & -485.64 & -245.06 & -236.68 & -470.10 & -480.91 & -486.40 & -479.42 & -481.78 & -467.63919 \\
\hline 22 & -532.90 & -727.94 & -559.09 & -248.76 & -247.35 & -538.41 & -527.98 & -556.60 & -539.48 & -528.07 & -570.46594 \\
\hline 23 & -473.19 & -698.09 & -472.09 & -182.95 & -178.74 & -459.60 & -456.90 & -486.09 & -446.49 & -435.79 & -486.12245 \\
\hline 24 & -498.56 & -710.77 & -485.77 & -195.10 & -185.25 & -475.73 & -471.13 & -497.48 & -476.43 & -481.84 & -505.23963 \\
\hline 25 & -309.92 & -456.09 & -303.97 & -110.80 & -97.41 & -298.57 & -295.69 & -298.64 & -275.30 & -287.64 & -295.23155 \\
\hline Overall & -730.59 & -1177.56 & -681.86 & -268.31 & -249.34 & -695.18 & -694.29 & -694.92 & -682.04 & -660.29 & -680.36 \\
\hline
\end{tabular}

Sl.No*: Serial number and name same as Table 2.

From new model 1 constants, one can calculate melting temperature and melting enthalpies. The back calculations are a bit tricky and we need to use a root finding method to calculate melting temperature and then melting enthalpy is estimated. The calculated values are reported in Table 9. It is observed that the melting temperature is much lower than actual values (Table 1); whereas the melting enthalpies for few compounds (Compound numbers 9, 11, 20, and 24) are magnitude wise matching with the computed values reported in Table 1 (Jain et al. method). This disparity may be attributed to use of approximate empirical expression for the fugacity ratio for the development of the solubility expression. Probably, exact expression would give better results and this is out of the scope of the present work.

To illustrate the ability of the proposed models, solubility data of 1-amino-2,3-dimethyl9,10-anthraquinone in supercritical carbon dioxide were selected as illustrated in Figures 1-4, respectively. In another illustration, we selected Red 15 (1-amino-4-hydroxyanthraquinone) to show the goodness of the new model 1 and the new model 2 (Figures 5 and 6). In Figure 7, the global mean AARD\% values of all models is depicted. In terms of global mean AARD, the overall order for the ability correlating of the models is: new model $2>$ new model $1>$ Adachi and $\mathrm{Lu}>$ Garlapati and Madras $>$ Keshmiri et al. $>$ Chrastil $>$ Khansary et al. $>$ Bian et al. $>$ Mitra and Wilson $>$ SLE model $>$ Reddy et al. 
Table 9. Computed $T_{m}$ and $\Delta H_{2}^{m}$ from new model 1.

\begin{tabular}{cccccc}
\hline S1.No* & $N_{\mathbf{1}}$ & $N_{\mathbf{2}}$ & $\boldsymbol{N}_{\mathbf{3}}$ & $\mathbf{T m}(\mathbf{K})^{\mathbf{a}}$ & $-\boldsymbol{\Delta H}_{2}^{\mathbf{m}}(\mathbf{J} / \mathbf{m o l})^{\mathbf{b}}$ \\
\hline 1 & -123.22 & 5849.4 & 16.501 & 140.5761 & $46,711.00$ \\
2 & -22.28 & 491.4 & 1.197 & 26.78756 & 3976.00 \\
3 & -119.11 & 5355.7 & 16.39 & 140.8947 & $42,620.00$ \\
4 & -255.5 & 11861 & 36.544 & 181.0266 & $94,055.00$ \\
5 & 352.36 & -17338 & -53.119 & $\mathrm{NA}$ & $\mathrm{NA}$ \\
6 & -35.941 & 1222 & 4.032 & 63.66784 & 9723.90 \\
7 & -44.086 & 1418.5 & 5.251 & 63.68529 & $11,226.00$ \\
8 & -133.51 & 5959.7 & 18.268 & 136.2973 & $47,423.00$ \\
9 & -65.421 & 2831.4 & 8.549 & 113.3441 & $22,545.00$ \\
10 & -118.88 & 5233.1 & 16.522 & 140.9835 & $41,585.00$ \\
11 & -81.27 & 3572.1 & 10.94 & 125.9199 & $28,425.00$ \\
12 & -54.582 & 2282.9 & 7.074 & 105.5913 & $18,215.00$ \\
13 & -73.225 & 3236.9 & 9.889 & 128.3756 & $25,761.00$ \\
14 & -203.75 & 9484.9 & 29.17 & 183.3302 & $75,220.00$ \\
15 & -80.585 & 3362.6 & 11.087 & 123.8086 & $26,666.00$ \\
16 & 967.57 & -52133 & -140.54 & $\mathrm{NA}$ & $\mathrm{NA}$ \\
17 & -95.533 & 4234.2 & 12.097 & 109.262 & $33,795.00$ \\
18 & -114.86 & 5180 & 15.189 & 124.6076 & $41,299.00$ \\
19 & -141.35 & 6609 & 19.113 & 141.5514 & $52,723.00$ \\
20 & -78.114 & 3418.2 & 9.746 & 104.079 & $27,366.00$ \\
21 & -31.261 & 922.3 & 2.662 & 43.4634 & 7402.40 \\
22 & -148.99 & 6728 & 20.046 & 131.4074 & $53,603.00$ \\
23 & -128.93 & 5935.5 & 17.365 & 136.1121 & $47,327.00$ \\
24 & -67.609 & 2866.9 & 8.373 & 98.16466 & $22,930.00$ \\
25 & 28.875 & -2317.9 & -5.576 & $\mathrm{NA}$ & $\mathrm{NA}$ \\
\hline
\end{tabular}

Sl.No*: Serial number and name same as Table 2. ${ }^{a}$ Newton method is used to calculate the root. $N_{1}+\frac{N_{2}}{T_{m}}+$ $N_{3} \ln \left(T_{m}\right)=0 .{ }^{\mathrm{b}} \Delta H_{2}^{m}=\left(-N_{2}+N_{3} T_{m}\right) R$. NA: Not able evaluated the root hence not reported.

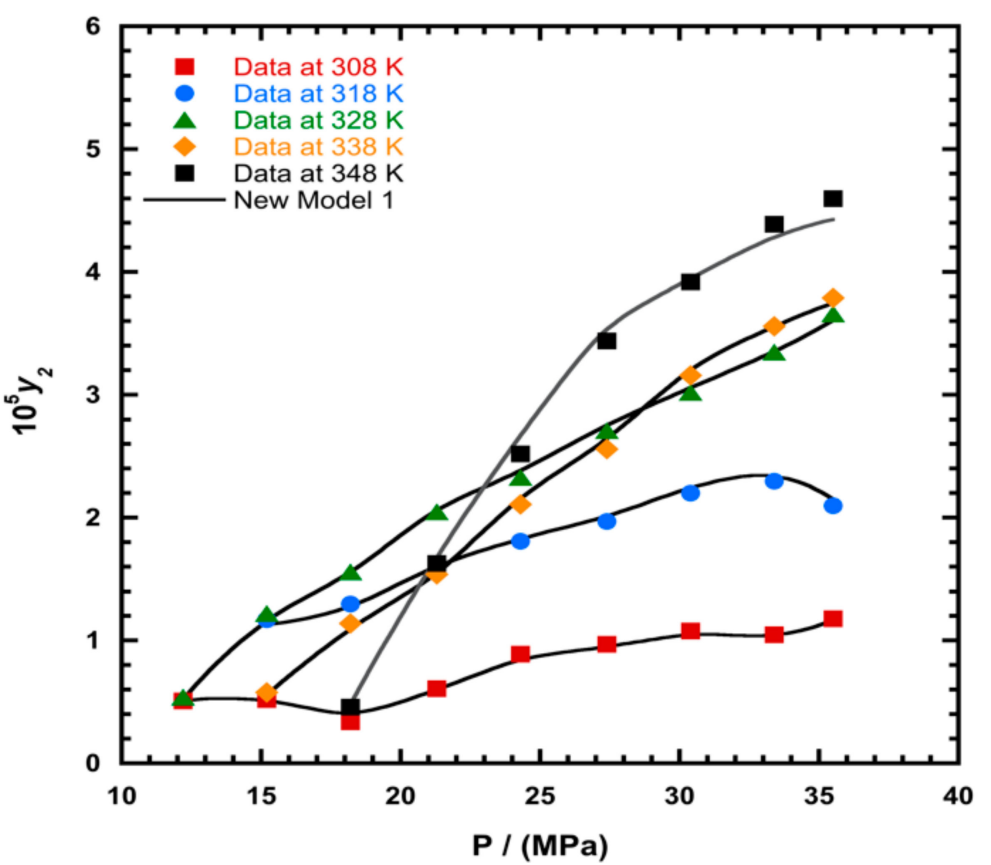

Figure 1. Plot of mole fraction $\left(y_{2}\right)$ as a function of pressure (P/MPa) for 1-amino-2,3-dimethyl-9,10anthraquinone, the solid line represents the proposed model 1 (Equation (18)). 


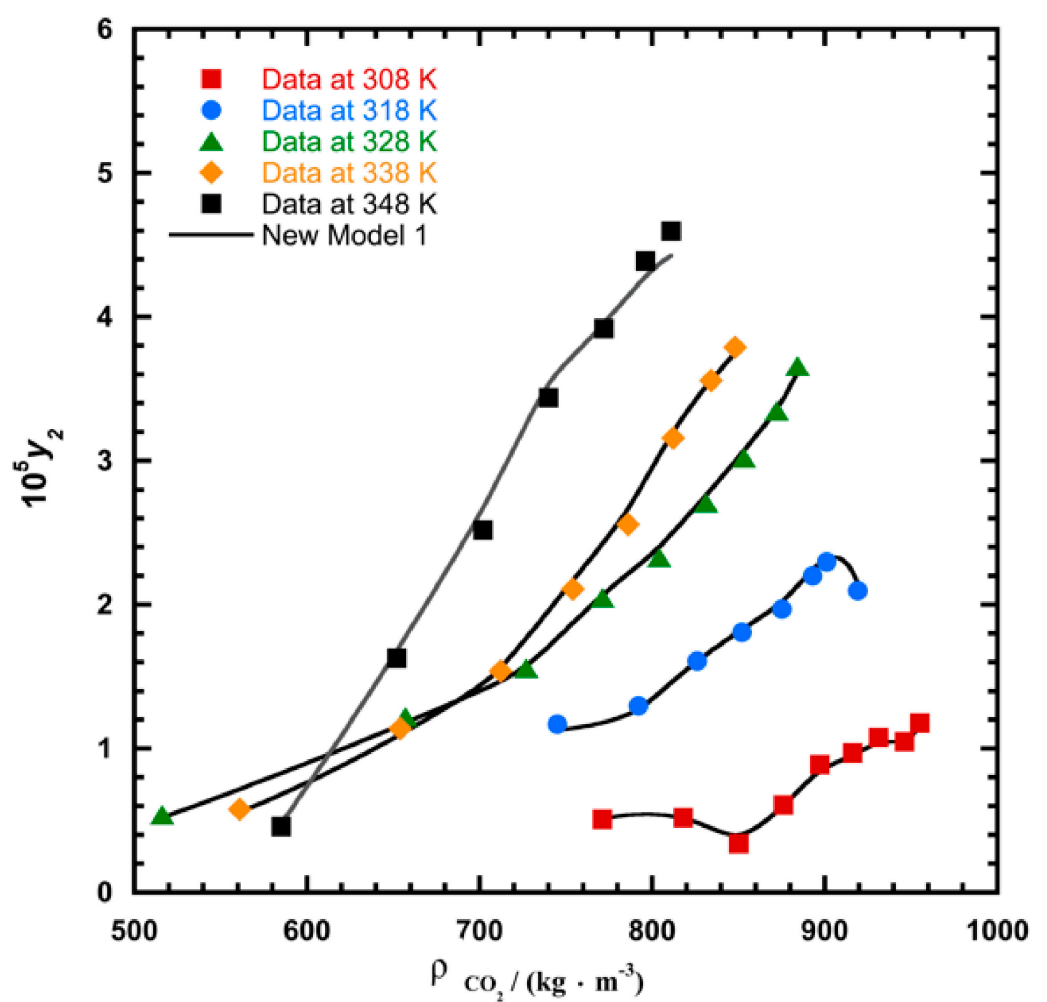

Figure 2. Plot of mole fraction $\left(y_{2}\right)$ as a function of density $\rho /\left(\mathrm{kg} \cdot \mathrm{m}^{-3}\right)$ for 1-amino-2,3-dimethyl9,10-anthraquinone, the solid line represents the proposed model 1 (Equation (18)).

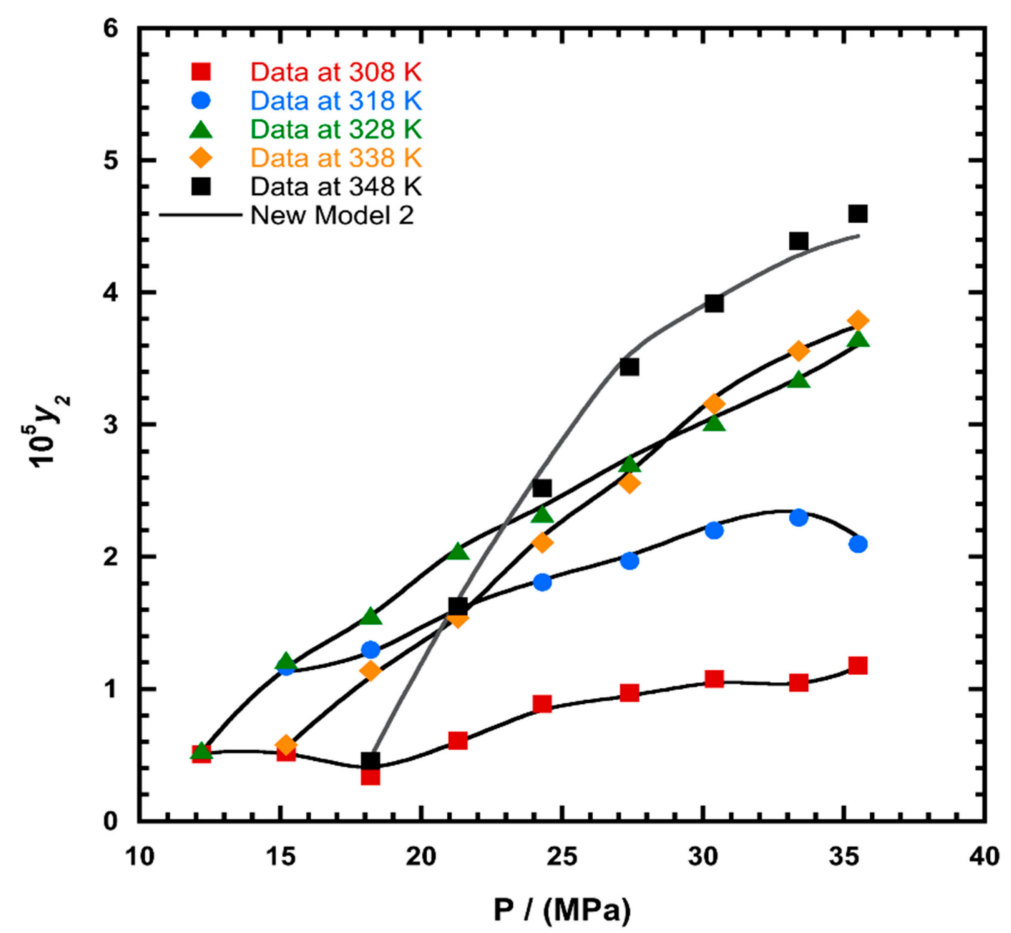

Figure 3. Plot of mole fraction $\left(y_{2}\right)$ as a function of pressure $(\mathrm{P} / \mathrm{MPa})$ for 1-amino-2,3-dimethyl-9,10anthraquinone, the solid line represents the proposed model 2 (Equation (21)). 


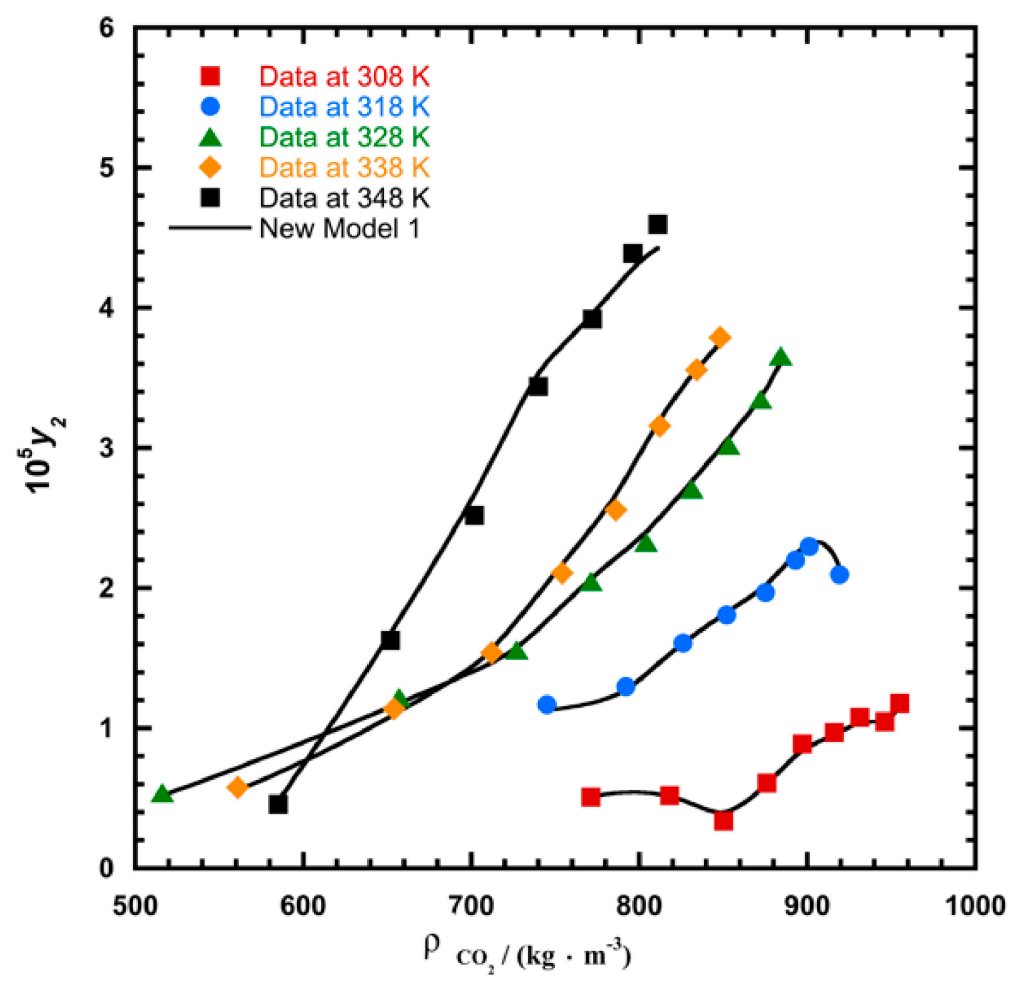

Figure 4. Plot of mole fraction $\left(y_{2}\right)$ as a function of density $\rho /\left(\mathrm{kg} \cdot \mathrm{m}^{-3}\right)$ for 1-amino-2,3-dimethyl9,10-anthraquinone, the solid line represents the proposed model 2 (Equation (21)).

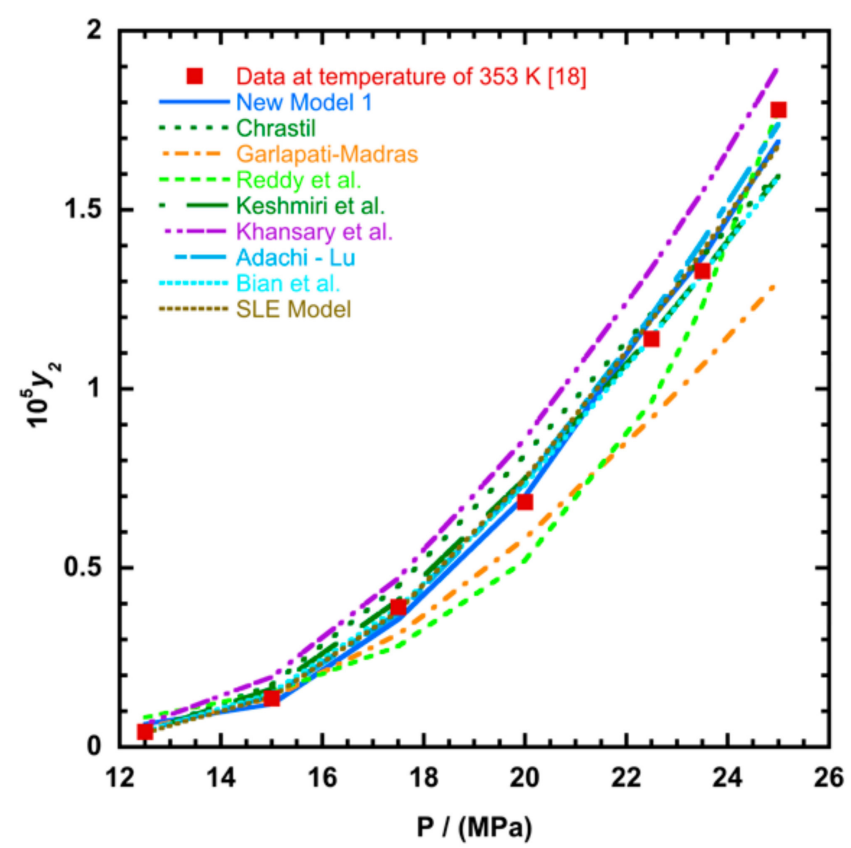

Figure 5. Plot of mole fraction solubility of Red 15 (1-amino-4-hydroxyanthraquinone) against pressure $(\mathrm{P} / \mathrm{MPa})$, the solid line presents the new model 1 (Equation (18)). 


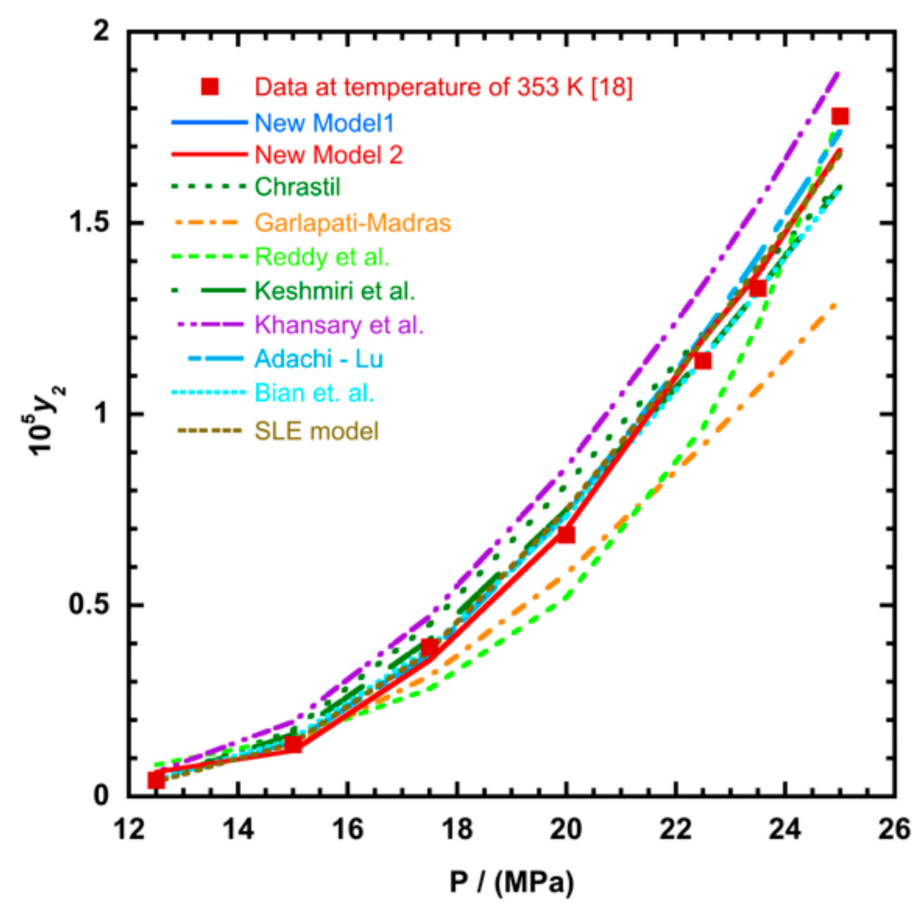

Figure 6. Plot of mole fraction solubility of Red 15 (1-amino-4-hydroxyanthraquinone) against pressure $(\mathrm{P} / \mathrm{MPa})$, the solid lines present the new model 1 (Equation (18)) and the new model 2 (Equation (21)), respectively.

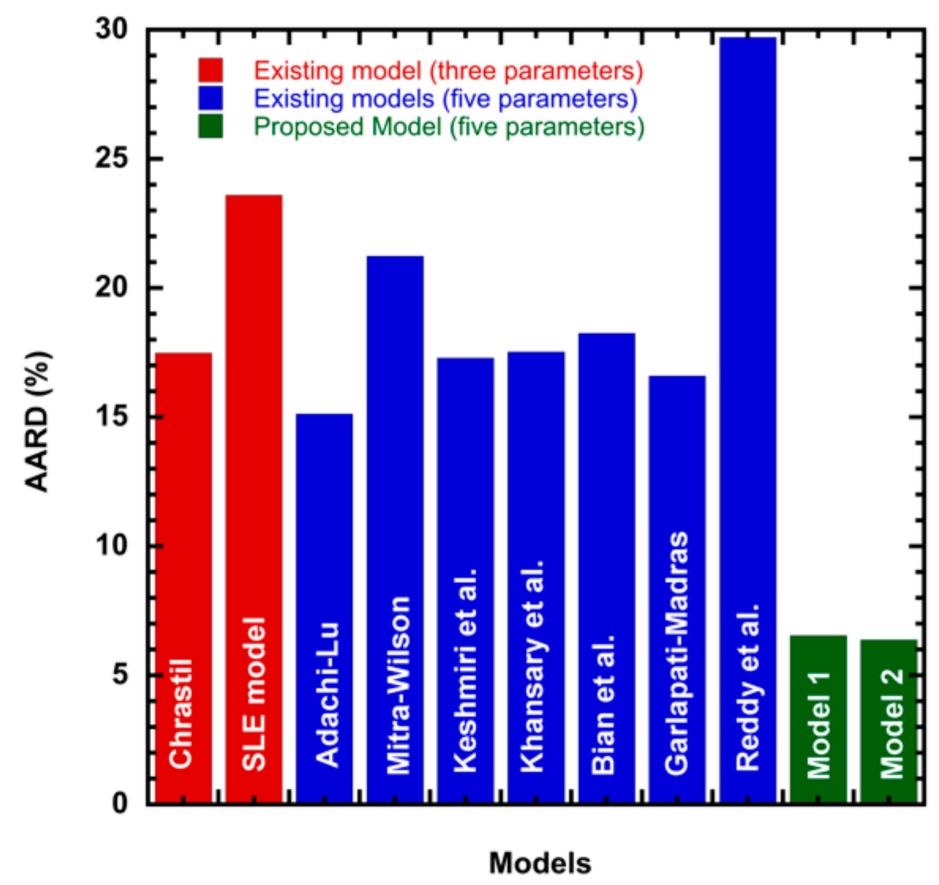

Figure 7. Global mean AARD\% of literature models and the proposed model.

\section{Conclusions}

The new models developed in this study may be useful in correlating solubility data of any compound in supercritical fluid. A comparison between the proposed models and some specific literature models (particularly three and five parameters constants) was made to correlate the solubility of 25 anthraquinone derivatives. The results showed that the proposed models exhibited excellent agreement with those experimental data in the literature and that the proposed models are superior to all of the other models considered 
in the present work with AARD of $6.538 \%$ for new model 1, and AARD of $6.377 \%$ for new model 2 . The new models of this work can be used for modeling solubility of any other system.

Supplementary Materials: The following are available online, Table S1: Correlation Constants of Chrastil's model, Table S2: Correlation Constants of Adachi-Lu model, Table S3: Correlation Constants of Mitra -Wilson model, Table S4: Correlation Constants of Keshmiri et al. model, Table S5: Correlation Constants of Khansary et al. model, Table S6: Correlation Constants of Bian et al. model, Table S7: Correlation Constants of Garlapati - Madras model, Table S8: Correlation Constants of Reddy et al. model.

Author Contributions: Conceptualization, R.S.A. and C.G.; methodology, R.S.A., C.G., and K.T.; software, R.S.A. and C.G.; validation, R.S.A., C.G., and K.T.; investigation, R.S.A.; writing-original draft preparation, R.S.A. and C.G.; writing—review and editing, R.S.A., C.G., and K.T.; supervision, C.G., and K.T. All authors have read and agreed to the published version of the manuscript.

Funding: This research received funding from Directorate of Research and Community Services, Directorate General of Strengthening Research and Development, Ministry of Research Technology and Higher Education, Republic Indonesia.

Data Availability Statement: Datas are available from the authors.

Acknowledgments: Research Grant sponsored by Directorate of Research and Community Services, Directorate General of Strengthening Research and Development, Ministry of Research Technology and Higher Education, Republic Indonesia (No. B/87/E3/RA.00/2020).

Conflicts of Interest: The authors declare no competing interests.

\section{References}

1. Gupta, R.B.; Shim, J.-J. Solubility in Supercritical Carbon Dioxide, 1st ed.; CRC Press: Boca Raton, FL, USA, 2007 ; ISBN 1420005995.

2. Garlapati, C.; Madras, G. New empirical expressions to correlate solubilities of solids in supercritical carbon dioxide. Thermochim. Acta 2010, 500, 123-127. [CrossRef]

3. Sodeifian, G.; Garlapati, C.; Hazaveie, S.M.; Sodeifian, F. Solubility of 2,4,7-Triamino-6-phenylpteridine (Triamterene, Diuretic Drug) in Supercritical Carbon Dioxide: Experimental Data and Modeling. J. Chem. Eng. Data 2020, 65, 4406-4416. [CrossRef]

4. Alwi, R.S.; Tamura, K. Measurement and Correlation of Derivatized Anthraquinone Solubility in Supercritical Carbon Dioxide. J. Chem. Eng. Data 2015, 60, 3046-3052. [CrossRef]

5. Kramer, A.; Thodos, G. Solubility of 1-hexadecanol and palmitic acid in supercritical carbon dioxide. J. Chem. Eng. Data 1988, 33, 230-234. [CrossRef]

6. Iwai, Y.; Koga, Y.; Fukuda, T.; Arai, Y. Correlation of Solubilities of High-Boiling Components in Supercritical Carbon Dioxide Using a Solution Model. J. Chem. Eng. Jpn. 1992, 25, 757-760. [CrossRef]

7. Su, C.-S.; Chen, Y.-P. Correlation for the solubilities of pharmaceutical compounds in supercritical carbon dioxide. Fluid Phase Equilib. 2007, 254, 167-173. [CrossRef]

8. Chrastil, J. Solubility of solids and liquids in supercritical gases. J. Phys. Chem. 1982, 86, 3016-3021. [CrossRef]

9. Sridar, R.; Bhowal, A.; Garlapati, C. A new model for the solubility of dye compounds in supercritical carbon dioxide. Thermochim. Acta 2013, 561, 91-97. [CrossRef]

10. Adachi, Y.; Lu, B.C.-Y. Supercritical fluid extraction with carbon dioxide and ethylene. Fluid Phase Equilib. 1983, 14, 147-156. [CrossRef]

11. Mitra, S.; Wilson, N.K. An Empirical Method to Predict Solubility in Supercritical Fluids. J. Chromatogr. Sci. 1991, 29, 305-309. [CrossRef]

12. Keshmiri, K.; Vatanara, A.; Yamini, Y. Development and evaluation of a new semi-empirical model for correlation of drug solubility in supercritical $\mathrm{CO}_{2}$. Fluid Phase Equilib. 2014, 363, 18-26. [CrossRef]

13. Asgarpour Khansary, M.; Amiri, F.; Hosseini, A.; Hallaji Sani, A.; Shahbeig, H. Representing solute solubility in supercritical carbon dioxide: A novel empirical model. Chem. Eng. Res. Des. 2015, 93, 355-365. [CrossRef]

14. Bian, X.-Q.; Zhang, Q.; Du, Z.-M.; Chen, J.; Jaubert, J.-N. A five-parameter empirical model for correlating the solubility of solid compounds in supercritical carbon dioxide. Fluid Phase Equilib. 2016, 411, 74-80. [CrossRef]

15. Reddy, T.A.; Srividya, R.; Garlapati, C. A new empirical model to correlate solubility of pharmaceutical compounds in supercritical carbon dioxide. J. Appl. Sci. Eng. Methodol. 2018, 4, 575-590.

16. Alwi, R.S.; Tanaka, T.; Tamura, K. Measurement and correlation of solubility of anthraquinone dyestuffs in supercritical carbon dioxide. J. Chem. Thermodyn. 2014, 74. [CrossRef]

17. Tamura, K.; Alwi, R.S.; Tanaka, T.; Shimizu, K. Solubility of 1-aminoanthraquinone and 1-nitroanthraquinone in supercritical carbon dioxide. J. Chem. Thermodyn. 2017, 104. [CrossRef] 
18. Tamura, K.; Alwi, R.S. Solubility of anthraquinone derivatives in supercritical carbon dioxide. Dye. Pigment. 2015, 113. [CrossRef]

19. Tamura, K.; Fukamizu, T. Representation of solubilities of phenylthioanthraquinone in supercritical carbon dioxide using Hansen solubility parameter. Fluid Phase Equilib. 2019, 489, 68-74. [CrossRef]

20. Jain, A.; Yang, G.; Yalkowsky, S.H. Estimation of Melting Points of Organic Compounds. Ind. Eng. Chem. Res. 2004, 43, 7618-7621. [CrossRef]

21. Fedors, R.F. A method for estimating both the solubility parameters and molar volumes of liquids. Polym. Eng. Sci. 1974, 14, 147-154. [CrossRef]

22. Giddings, J.C.; Myers, M.N.; McLaren, L.; Keller, R.A. High Pressure Gas Chromatography of Nonvolatile Species. Science 1968, 162, 67-73. [CrossRef] [PubMed]

23. National Institute of Standards and Technology. U.S. Department of Commerce. Available online: https://webbook.nist.gov/ chemistry (accessed on 28 September 2020).

24. Prausnitz, J.M.; Lichtenthaler, R.N.; de Azvedo, E.G. Molecular Thermodynamics of Fluid-Phase Equilibria, 3rd ed.; Prentice-Hall: Upper Saddle River, NJ, USA, 1999.

25. Nordström, F.L.; Rasmuson, Å.C. Solubility and Melting Properties of Salicylic Acid. J. Chem. Eng. Data 2006, 51, 1668-1671. [CrossRef]

26. Kramer, A.; Thodos, G. Adaptation of the Flory-Huggins theory for modeling supercritical solubilities of solids. Ind. Eng. Chem. Res. 1988, 27, 1506-1510. [CrossRef]

27. Lee, J.W.; Min, J.M.; Bae, H.K. Solubility Measurement of Disperse Dyes in Supercritical Carbon Dioxide. J. Chem. Eng. Data 1999, 44, 684-687. [CrossRef]

28. Lagarias, J.C.; Reeds, J.A.; Wright, M.H.; Wright, P.E. Convergence Properties of the Nelder-Mead Simplex Method in Low Dimensions. SIAM J. Optim. 1998, 9, 112-147. [CrossRef]

29. Reddy, T.A.; Garlapati, C. Dimensionless Empirical Model to Correlate Pharmaceutical Compound Solubility in Supercritical Carbon Dioxide. Chem. Eng. Technol. 2019, 42, 2621-2630. [CrossRef]

30. Akaike, H. Likelihood of a model and information criteria. J. Econom. 1981, 16, 3-14. [CrossRef]

31. Akaike, H. A New Look at the Statistical Model Identification. IEEE Trans. Automat. Contr. 1974, 19, 716-723. [CrossRef]

32. Joung, S.N.; Shin, H.Y.; Park, Y.H.; Yoo, K.-P. Measurement and correlation of solubility of disperse anthraquinone and azo dyes in supercritical carbon dioxide. Korean J. Chem. Eng. 1998, 15, 78-84. [CrossRef]

33. Lee, J.W.; Park, M.W.; Bae, H.K. Measurement and correlation of dye solubility in supercritical carbon dioxide. Fluid Phase Equilib. 2000, 173, 277-284. [CrossRef]

34. Coelho, J.P.; Stateva, R.P. Solubility of red 153 and blue 1 in supercritical carbon dioxide. J. Chem. Eng. Data 2011, 56, 4686-4690. [CrossRef]

35. Ferri, A.; Banchero, M.; Manna, L.; Sicardi, S. An experimental technique for measuring high solubilities of dyes in supercritical carbon dioxide. J. Supercrit. Fluids 2004, 30, 41-49. [CrossRef]

36. Fat'hi, M.R.; Yamini, Y.; Sharghi, H.; Shamsipur, M. Solubilities of Some 1,4-Dihydroxy-9,10-anthraquinone Derivatives in Supercritical Carbon Dioxide. J. Chem. Eng. Data 1998, 43, 400-402. [CrossRef]

37. Shamsipur, M.; Karami, A.R.; Yamini, Y.; Sharghi, H. Solubilities of Some Aminoanthraquinone Derivatives in Supercritical Carbon Dioxide. J. Chem. Eng. Data 2003, 48, 71-74. [CrossRef]

38. Shamsipur, M.; Karami, A.R.; Yamini, Y.; Sharghi, H. Solubilities of some 1-hydroxy-9,10-anthraquinone derivatives in supercritical carbon dioxide. J. Supercrit. Fluids 2004, 32, 47-53. [CrossRef] 